\title{
Connection matrices and Lie algebra weight systems for multiloop chord diagrams
}

\author{
Alexander Schrijver ${ }^{1}$
}

Received: 22 September 2014 / Accepted: 23 May 2015 / Published online: 13 June 2015

C The Author(s) 2015. This article is published with open access at Springerlink.com

\begin{abstract}
We give necessary and sufficient conditions for a weight system on multiloop chord diagrams to be obtainable from a metrized Lie algebra representation, in terms of a bound on the ranks of associated connection matrices. Here a multiloop chord diagram is a graph with directed and undirected edges so that at each vertex, precisely one directed edge is entering and precisely one directed edge is leaving, and each vertex is incident with precisely one undirected edge. Weight systems on multiloop chord diagrams yield the Vassiliev invariants for knots and links. The $k$-th connection matrix of a function $f$ on the collection of multiloop chord diagrams is the matrix with rows and columns indexed by $k$-labeled chord tangles and with entries equal to the $f$ value on the join of the tangles.
\end{abstract}

Keywords Multiloop chord diagram - Weight system · Metrized Lie algebra · Connection matrix

Mathematics Subject Classification $\quad 05 \mathrm{E} 15 \cdot 17 \mathrm{~B} 10 \cdot 57 \mathrm{M} 27$

\section{Introduction}

In this introduction, we describe our results for those familiar with the basic theory of weight systems on chord diagrams (cf. [4]). In the next section, we define concepts, so as to fix terminology and so as to make the paper self-contained also for those not familiar with weight systems.

Alexander Schrijver

lex@cwi.nl

1 Korteweg-de Vries Institute for Mathematics, University of Amsterdam,

Science Park 105, 1098 XG Amsterdam, The Netherlands 
Bar-Natan [1,2] and Kontsevich [10] have shown that any finite-dimensional representation $\rho$ of a metrized Lie algebra $\mathfrak{g}$ yields a weight system $\varphi_{\mathfrak{g}}^{\rho}$ on chord diagrams - more generally on multiloop chord diagrams. (These are chord diagrams in which more than one Wilson loop is allowed. Weight systems on multiloop chord diagrams yield Vassiliev link invariants.)

In this paper, we characterize the weight systems that arise this way. More precisely, we show the equivalence of the following conditions for any complex-valued weight system $f$ :

(i) $f=\varphi_{\mathfrak{g}}^{\rho}$ for some completely reducible faithful representation $\rho$ of some metrized Lie algebra $\mathfrak{g}$;

(ii) $f=\varphi_{\mathfrak{g}}^{\rho}$ for some representation $\rho$ of some metrized Lie algebra $\mathfrak{g}$;

(iii) $f$ is the partition function $p_{R}$ of some $n \in \mathbb{Z}_{+}$and $R \in S^{2}(\mathfrak{g l}(n))$;

(iv) $f(\bigcirc) \in \mathbb{R}$ and $\operatorname{rank}\left(M_{f, k}\right) \leq f(\bigcirc)^{2 k}$ for each $k$.

Throughout, $\mathfrak{g l}(n)=\mathfrak{g l}(n, \mathbb{C})$, while $\mathbb{C}$ may be replaced by any algebraically closed field of characteristic 0 . All representations are assumed to be finite-dimensional. In (i), the Lie algebra $\mathfrak{g}$ is necessarily reductive. The largest part of the proof consists of showing (iv) $\Longrightarrow$ (iii).

We give some explanation of the conditions (iii) and (iv). First, $S^{2}(\mathfrak{g l}(n))$ denotes the space of tensors in $\mathfrak{g l}(n) \otimes \mathfrak{g l}(n)$ that are symmetric (i.e., invariant under the linear function induced by $X \otimes Y \rightarrow Y \otimes X)$. The partition function $p_{R}$ of $R \in S^{2}(\mathfrak{g l}(n))$ can be intuitively described as the function on multiloop chord diagrams obtained by inserting a copy of the tensor $R$ at each chord, assigning ('multilinearly') its two tensor components in $\mathfrak{g l}(n)$ to the two ends of that chord, next calculating, along any Wilson loop, the trace of the product of the elements in $\mathfrak{g l}(n)$ assigned to the vertices of that Wilson loop (in order), and finally taking the product of these traces over all Wilson loops. (This is in analogy to the partition function of the 'vertex model' in de la Harpe and Jones [5].)

In (1) (iv), $\bigcirc$ is the chord diagram without chords. To describe the matrix $M_{f, k}$, we need ' $k$-labeled multiloop chord tangles' or ' $k$-tangles' for short. A $k$-tangle is a multiloop chord diagram with $k$ directed edges entering it, labeled $1, \ldots, k$, and $k$ directed edges leaving it, also labeled $1, \ldots, k$, like the 4 -tangle

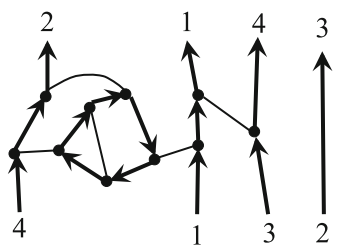

Let $\mathcal{T}_{k}$ denote the collection of all $k$-tangles. For $S, T \in \mathcal{T}_{k}$, let $S \cdot T$ be the multiloop chord diagram obtained by glueing $S$ and $T$ appropriately together: $S \cdot T$ arises from the disjoint union of $S$ and $T$ by identifying outgoing edge labeled $i$ of $S$ with ingoing edge labeled $i$ of $T$, and similarly, identifying outgoing edge labeled $i$ of $T$ with 
ingoing edge labeled $i$ of $S$ (for $i=1, \ldots, k$ ). Then the $k$-th connection matrix $M_{f, k}$ of $f$ is the $\mathcal{T}_{k} \times \mathcal{T}_{k}$ matrix with entry $f(S \cdot T)$ in position $(S, T) \in \mathcal{T}_{k} \times \mathcal{T}_{k}$. (Studying such matrices roots in work of Freedman et al. [7] and Szegedy [14], cf. also the recent book by Lovász [12].)

The implications (i) $\Longrightarrow$ (ii) $\Longrightarrow$ (iii) $\Longrightarrow$ (iv) are easy-the content of this paper is proving the reverse implications. Indeed, (i) $\Longrightarrow$ (ii) is trivial. To see (ii) $\Longrightarrow$ (iii), recall the fundamental construction of Bar-Natan [1,2] and Kontsevich [10]. Let $\mathfrak{g}$ be a metrized Lie algebra, and let $\rho: \mathfrak{g} \rightarrow \mathfrak{g l}(n)$ be a representation. Let $b_{1}, \ldots, b_{k}$ be any orthonormal basis of $\mathfrak{g}$ and define

$$
R(\mathfrak{g}, \rho):=\sum_{i=1}^{k} \rho\left(b_{i}\right) \otimes \rho\left(b_{i}\right) \in S^{2}(\mathfrak{g l}(n))
$$

(which is independent of the choice of the orthonormal basis). Then $\phi_{\mathfrak{g}}^{\rho}:=p_{R(\mathfrak{g}, \rho)}$ is a weight system. So one has (ii) $\Longrightarrow$ (iii).

The Lie bracket is not involved in condition (1) (iii), and it is required only that $p_{R}$ be a weight system. Indeed, not each $R \in S^{2}(\mathfrak{g l}(n))$ for which $p_{R}$ is a weight system arises as above from a Lie algebra. For instance, let $B_{1}:=\left(\begin{array}{ll}1 & 1 \\ 0 & 0\end{array}\right)$ and $B_{2}:=\left(\begin{array}{ll}0 & 1 \\ 0 & 1\end{array}\right)$ (as elements of $\mathfrak{g l}(2))$, and set $R:=B_{1}^{\otimes 2}+B_{2}^{\otimes 2} \in S^{2}(\mathfrak{g l}(2))$. Then $p_{R}$ is identically two on connected diagrams; hence, $p_{R}$ is a weight system, but there is no representation $\rho$ of a metrized Lie algebra $\mathfrak{g}$ with $R=R(\mathfrak{g}, \rho)$ (essentially because the matrices $B_{1}$ and $B_{2}$ do not span a matrix Lie algebra).

The implication (iii) $\Longrightarrow$ (iv) follows from the fact that for any $k$ and any $k$-tangles $S$ and $T, p_{R}(S \cdot T)$ can be described as the trace of the product of certain elements $\widehat{p}_{R}(S)$ and $\widehat{p}_{R}(T)$ of $\mathfrak{g l}(n)^{\otimes k}$, where the latter space has dimension $n^{2 k}$.

Our proof of the reverse implications is based on some basic results of algebraic geometry (Nullstellensatz), invariant theory (first and second fundamental theorem, closed orbit theorem), and (implicitly through [13]) the representation theory of the symmetric group. It consists of showing that if (1) (iv) is satisfied, then $n:=f(\bigcirc)$ belongs to $\mathbb{Z}_{+}$and the affine $\operatorname{GL}(n)$-variety

$$
\mathcal{V}:=\left\{R \in S^{2}(\mathfrak{g l}(n)) \mid p_{R}=f\right\}
$$

is nonempty (which is (iii)), and each $R$ in the (unique) closed GL(n)-orbit in $\mathcal{V}$ produces a completely reducible faithful representation of a Lie algebra as in (i).

We must emphasize here that the above will be proved for multiloop chord diagrams. We do not know in how far it remains true when restricting the functions to ordinary, one-loop, chord diagrams.

We also do not know in how far the Lie algebra $\mathfrak{g}$ and the representation $\rho$ in (1) (i) are unique (up to the action of $\operatorname{GL}(n)$ where $n$ is the dimension of $\rho$ ), although the existence is shown by construction from the unique closed GL $(n)$-orbit in $\mathcal{V}$. A partial result in this direction was given by Kodiyalam and Raghavan [9]: let $\mathfrak{g}$ and $\mathfrak{g}^{\prime}$ be $n$-dimensional semisimple Lie algebras, with the Killing forms as metrics, and let $\rho$ and $\rho^{\prime}$ be the adjoint representations; if $\varphi_{\mathfrak{g}}^{\rho}=\varphi_{\mathfrak{g}^{\prime}}^{\rho^{\prime}}$ on (one-loop) chord diagrams, then $\mathfrak{g}=\mathfrak{g}^{\prime}$. 


\section{Preliminaries}

\subsection{Multiloop chord diagrams and weight systems}

A multiloop chord diagram is a cubic graph $C$ in which a collection of disjoint oriented cycles is specified that cover all vertices. These cycles are called the Wilson loops, and the remaining edges (that form a perfect matching on the vertex set of $C$ ) are called the chords.

Alternatively, a multiloop chord diagram can be described as a graph with directed and undirected edges such that for each vertex $v$ :

$v$ is entered by precisely one directed edge, is left by precisely one directed edge,

and is incident with precisely one undirected edge,

as in
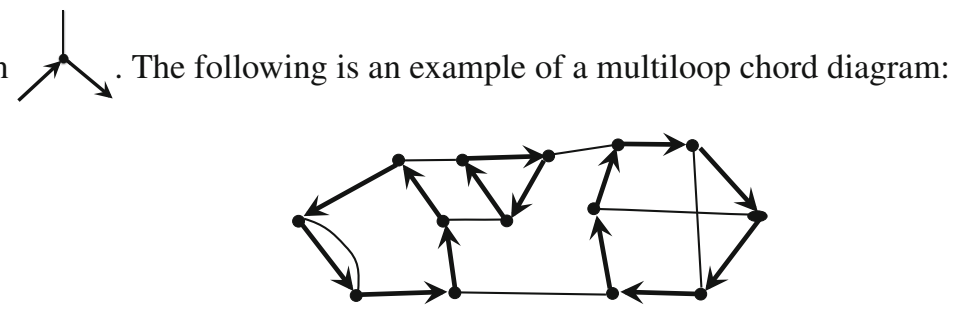

Directed loops are allowed, but no undirected loops. Moreover, we allow the 'vertexless directed loop' $\bigcirc$ (in other words, the chord diagram of order 0 )—-more precisely, components of a multiloop chord diagram may be vertexless directed loops.

Let $\mathcal{C}$ denote the collection of multiloop chord diagrams. Basic for Vassiliev knot invariants (cf. [4]) is functions $f$ on $\mathcal{C}$ that satisfy certain linear relations, called the 4-term (4T) relations. They can be visualized as:

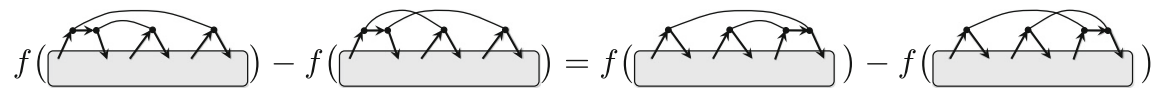

(Each of the four gray rectangles contains the rest of the diagram, the same in each rectangle.)

Functions satisfying the 4T relations are called weight systems. More precisely, we call a function $f$ on multiloop chord diagrams a weight system if it satisfies the 4T relations, and moreover it is multiplicative: $f(\emptyset)=1$ and $f(C \sqcup D)=f(C) f(D)$ for all multiloop chord diagrams $C, D$, where $C \sqcup D$ denotes the disjoint union of $C$ and $D$. Hence, any weight system is determined by its values on connected multiloop chord diagrams.

Through the Kontsevich integral, each $\mathbb{C}$-valued weight system on the collection of multiloop chord diagram with some fixed number of chords and some fixed number $t$ of Wilson loops gives an invariant for links with $t$ components. They produce precisely the Vassiliev invariants for knots and links. We refer for these important concepts to the book of Chmutov, Duzhin, Mostovoy [4] — for understanding our treatment below they are, however, not needed. 


\subsection{Some notation and linear algebra}

As usual,

$$
\begin{aligned}
\mathbb{Z}_{+}:= & \text {the set of nonnegative integers, and }[k]:=\{1, \ldots, k\} \\
& \text { for any } k \in \mathbb{Z}_{+} .
\end{aligned}
$$

For any set $\mathcal{X}, \mathbb{C} \mathcal{X}$ denotes the linear space of formal $\mathbb{C}$-linear combinations of finitely many elements of $\mathcal{X}$. (Occasionally, elements of $\mathbb{C} \mathcal{X}$ are called quantum elements of $\mathcal{X}$.) Any function on $\mathcal{X}$ to a $\mathbb{C}$-linear space can be uniquely extended to a linear function on $\mathbb{C} \mathcal{X}$.

For a linear space $X, S^{2}(X)$ denotes the space of symmetric elements of $X \otimes X$, i.e., those invariant under the linear operation on $X \otimes X$ induced by $x \otimes y \mapsto y \otimes x$. It is elementary matrix theory to prove that if $X$ is finite-dimensional, then for any $R \in S^{2}(X)$ there is a unique subspace $Y$ of $X$ and a unique nondegenerate bilinear form on $Y$ such that for each orthonormal basis $b_{1}, \ldots, b_{k}$ of $Y$ one has

$$
R=\sum_{i=1}^{k} b_{i} \otimes b_{i}
$$

Considering $R$ as matrix, $Y$ is equal to the column space of $R$.

\subsection{Partition functions}

Each $R \in S^{2}(\mathfrak{g l}(n))$ gives a function $p_{R}$ on multiloop chord diagrams as follows. Fix a basis of $\mathbb{C}^{n}$, and write $R=\left(R_{i, j}^{k, l}\right)$, with $i, j, k, l \in[n]$. Then the partition function $p_{R}: \mathcal{C} \rightarrow \mathbb{C}$ is given by

$$
p_{R}(C):=\sum_{\varphi: A \rightarrow[n]} \prod_{u v \in E} R_{\varphi\left(u_{\text {in }}\right), \varphi\left(v_{\text {in }}\right)}^{\varphi\left(u_{\text {out }}\right)}
$$

for any multiloop chord diagram $C$, where $A$ and $E$ denote the sets of directed and undirected edges, respectively, of $C$ and where $v_{\text {in }}$ and $v_{\text {out }}$ denote the ingoing and the outgoing directed edge, respectively, at a vertex $v$. This implies $p_{R}(\bigcirc)=n$. Note that (7) is independent of the basis of $\mathbb{C}^{n}$ chosen.

We will also write $p(C)(R)$ for $p_{R}(C)$. Then $p(C): S^{2}(\mathfrak{g l}(n)) \rightarrow \mathbb{C}$ is $\mathrm{GL}(n)$ invariant. (Throughout, GL $(n)$ acts on $\mathfrak{g l}(n)$ by $h \cdot M:=h M h^{-1}$ for $h \in \operatorname{GL}(n)$ and $M \in \mathfrak{g l}(n)$.) By the First Fundamental Theorem (FFT) of invariant theory (cf. [8] Corollary 5.3.2), each GL(n)-invariant regular function $S^{2}(\mathfrak{g l}(n)) \rightarrow \mathbb{C}$ is a linear combination of functions $p(C)$ with $C$ a multiloop chord diagram. (Here multiloop is essential.)

It will be convenient to notice at this point the following alternative description of the partition function $p_{R}$. Let $b_{1}, \ldots, b_{k} \in \mathfrak{g l}(n)$ be as in (6), with $X:=\mathfrak{g l}(n)$. Let $C$ be a multiloop chord diagram. Consider a function $\psi: E \rightarrow[k]$. 'Assign' matrix 
$b_{\psi(u v)}$ to each of the ends $u$ and $v$ of any undirected edge $u v$. Each of the Wilson loops in $C$ now has matrices assigned to its vertices, and on each Wilson loop, we can take the trace of the product of these matrices (in order). Taking the product of these traces over all Wilson loops, and next summing up these products over all $\psi: E \rightarrow[k]$, gives $p_{R}(C)$. (In the idiom of Szegedy [14], we here color the undirected edges, with $k$ colors, while in (7) we color the directed edges, with $n$ colors).

\subsection{Tangles}

We need an extension of the concept of multiloop chord diagram. Define a multiloop chord tangle, or tangle for short, as a graph with directed and undirected edges, such that each vertex $v$ either satisfies (4) or $v$ is incident with precisely one directed edge and with no undirected edge. Of the latter type of vertex, there are two kinds: vertices, called roots, with one outgoing edge, and vertices, called sinks, with one ingoing edge. The numbers of roots and of sinks are necessarily equal. Again, a tangle may have components that are just the vertexless directed loop $\bigcirc$.

A $k$-labeled multiloop chord tangle, or just $k$-tangle, is a tangle with precisely $k$ roots, equipped with labels $1, \ldots, k$, and $k$ sinks, also equipped with labels $1, \ldots, k$. Denote the collection of $k$-tangles by $\mathcal{T}_{k}$. So $\mathcal{T}_{0}=\mathcal{C}$.

For $S, T \in \mathcal{T}_{k}$, let $S \cdot T$ be the multiloop chord diagram arising from the disjoint union of $S$ and $T$ by, for each $i=1, \ldots, k$, identifying the $i$-labeled $\operatorname{sink}$ in $S$ with the $i$-labeled root in $T$, and identifying the $i$-labeled root in $S$ with the $i$-labeled $\operatorname{sink}$ in $T$; after each identification, we ignore identified points as vertex, joining its two incident directed edges into one directed edge; that is, $\longrightarrow$ - $\longrightarrow$ becomes $\longrightarrow$. Note that this operation may introduce vertexless loops. We extend this operation bilinearly to $\mathbb{C} \mathcal{T}_{k}$. If $C, D \in \mathcal{C}=\mathcal{T}_{0}$, then $C \cdot D$ is equal to the disjoint union of $C$ and $D$.

Weight systems are determined by the $4 \mathrm{~T}$ 'quantum' 3 -tangle $\tau_{4}$, which is the element of $\mathbb{C} \mathcal{T}_{3}$ emerging from the 4T relations:

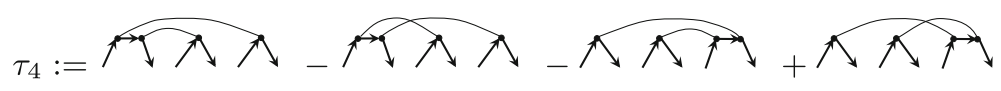

(We have omitted labels, as they are obvious (one may take labels 1, 1, 2, 2, 3, 3 from left to right in each tangle in (8)).) Thus, a function $f$ on $\mathcal{C}$ is a weight system if and only if $f\left(\tau_{4} \cdot T\right)=0$ for each 3-tangle $T$.

\subsection{The partition function on tangles}

We extend the function $p_{R}$ on multiloop chord diagrams to a function $\widehat{p}_{R}$ on tangles. For each $R \in S^{2}(\mathfrak{g l}(n))$ and $k \in \mathbb{Z}_{+}$, the partition function $\widehat{p}_{R}: \mathcal{T}_{k} \rightarrow \mathfrak{g l}(n)^{\otimes k}$ is defined as, for $C \in \mathcal{T}_{k}$ :

$$
\widehat{p}_{R}(C):=\sum_{\varphi: A \rightarrow[n]} \prod_{u v \in E} R_{\varphi\left(u_{\text {in }}\right), \varphi\left(v_{\text {in }}\right)}^{\varphi\left(u_{\text {out }}\right)} \bigotimes_{j=1}^{k} E_{\varphi\left(a_{j}\right)}^{\varphi\left(a_{j}^{*}\right)} .
$$


Here we use the same notation as for (7). Moreover, $a_{1}, \ldots, a_{k}$ are the directed edges leaving the roots labeled $1, \ldots, k$, respectively, and $a_{1}^{*}, \ldots, a_{k}^{*}$ are the directed edges entering the sinks labeled $1, \ldots, k$, respectively. For $h, i \in[n], E_{h}^{i}$ is the matrix in $\mathfrak{g l}(n)$ with 1 in position $(h, i)$ and 0 elsewhere. Note that (9) is independent of the basis of $\mathbb{C}^{n}$ chosen.

Again, set $\widehat{p}(C)(R):=\widehat{p}_{R}(C)$. Then $\widehat{p}(C): S^{2}(\mathfrak{g l}(n)) \rightarrow \mathfrak{g l}(n)^{\otimes k}$ is a $\operatorname{GL}(n)$ equivariant regular function, and each such function is a linear combination of functions $\widehat{p}(C)$ (by the FFT for invariant theory).

Note that $p_{R}$ is the restriction of $\widehat{p}_{R}$ to $\mathcal{C}$ and that

$$
p_{R}(S \cdot T)=\operatorname{tr}\left(\widehat{p}_{R}(S) \widehat{p}_{R}(T)\right)
$$

for all $k$-tangles $S$ and $T$ (under the natural identification $\mathfrak{g l}(n)^{\otimes k}=\operatorname{End}\left(\left(\mathbb{C}^{n}\right)^{\otimes k}\right)$ ).

\subsection{Weight systems and Lie algebras}

A Lie algebra $\mathfrak{g}$ is called metrized if it is equipped with a nondegenerate bilinear form $\langle.,$.$\rangle that is ad-invariant, i.e., satisfies \langle[x, y], z\rangle=\langle x,[y, z]\rangle$ for all $x, y, z \in \mathfrak{g}$.

For any $R \in S^{2}(\mathfrak{g l}(n))$, choose linearly independent $b_{1}, \ldots, b_{k} \in \mathfrak{g l}(n)$ such that $R=\sum_{i=1}^{k} b_{i} \otimes b_{i}$ (as in (6), taking $\left.X:=\mathfrak{g l}(n)\right)$. Then the following fundamental insight was given by Bar-Natan [1,2]:

$\widehat{p}_{R}\left(\tau_{4}\right)=0$ if and only if $b_{1}, \ldots, b_{k}$ form an orthonormal basis of a metrized

Lie algebra $\mathfrak{g} \subseteq \mathfrak{g l}(n)$.

In fact, if $\mathfrak{g}$ is a metrized Lie algebra and $\rho: \mathfrak{g} \rightarrow \mathfrak{g l}(n)$ is a representation, then $R:=$ $\sum_{i=1}^{k} \rho\left(b_{i}\right) \otimes \rho\left(b_{i}\right)$ satisfies $\widehat{p}_{R}\left(\tau_{4}\right)=0$ (where again $b_{1}, \ldots, b_{k}$ is any orthonormal basis of $\mathfrak{g})$. This implies that

$$
\varphi_{\mathfrak{g}}^{\rho}:=p_{R}
$$

is a weight system.

\section{Theorem and proof}

Define, for any $f: \mathcal{C} \rightarrow \mathbb{C}$ and $k \in \mathbb{Z}_{+}$, the $\mathcal{T}_{k} \times \mathcal{T}_{k}$ matrix $M_{f, k}$ by

$$
\left(M_{f, k}\right)_{S, T}:=f(S \cdot T)
$$

for $S, T \in \mathcal{T}_{k}$. 
Theorem Let $f: \mathcal{C} \rightarrow \mathbb{C}$ be a weight system. Then the following are equivalent:

(i) $f=\varphi_{\mathfrak{g}}^{\rho}$ for some completely reducible faithful representation $\rho$ of some metrized Lie algebra $\mathfrak{g}$;

(ii) $f=\varphi_{\mathfrak{g}}^{\rho}$ for some representation $\rho$ of some metrized Lie algebra $\mathfrak{g}$;

(iii) $f$ is the partition function $p_{R}$ of some $n \in \mathbb{Z}_{+}$and $R \in S^{2}(\mathfrak{g l}(n))$;

(iv) $f(\bigcirc) \in \mathbb{R}$ and $\operatorname{rank}\left(M_{f, k}\right) \leq f(\bigcirc)^{2 k}$ for each $k$.

Proof (i) $\Longrightarrow$ (ii) is trivial, and (ii) $\Longrightarrow$ (iii) is easy by taking $R=\sum_{i=1}^{k} \rho\left(b_{i}\right) \otimes \rho\left(b_{i}\right)$ for some orthonormal basis $b_{1}, \ldots, b_{k}$ of $\mathfrak{g}$.

As to (iii) $\Longrightarrow$ (iv): $f(\bigcirc)=n \in \mathbb{R}$ is direct, while $\operatorname{rank}\left(M_{f, k}\right) \leq n^{2 k}$ follows from (10), since $\widehat{p}_{R}(S)$ and $\widehat{p}_{R}(T)$ belong to $\mathfrak{g l}(n)^{\otimes k}$, which is $n^{2 k}$-dimensional.

It remains to show (iv) $\Longrightarrow$ (i). For $k \in \mathbb{Z}_{+}$and $S, T \in \mathcal{T}_{k}$, define (next to the 'inner product' $S \cdot T$ ) the product $S T$ as the $k$-tangle obtained from the disjoint union of $S$ and $T$ by identifying sink labeled $i$ of $S$ with root labeled $i$ of $T$ and ignoring this vertex as vertex (i.e., $\longrightarrow$ - $\longrightarrow$ becomes $\longrightarrow$ ), for $i=1, \ldots, k$; the roots of $S$ labeled $1, \ldots, k$ and sinks of $T$ labeled $1, \ldots, k$ make $S T$ to a $k$-tangle again.

Clearly, this product is associative and satisfies $(S T) \cdot U=S \cdot(T U)$ for all $k$-tangles $S, T, U$. Moreover, there is a unit, denoted by $\mathbf{1}_{k}$, consisting of $k$ disjoint directed edges $e_{1}, \ldots, e_{k}$, where both ends of $e_{i}$ are labeled $i(i=1, \ldots, k)$.

Extend the product $S T$ bilinearly to $\mathbb{C} \mathcal{T}_{k}$, making $\mathbb{C} \mathcal{I}_{k}$ to a $\mathbb{C}$-algebra. Let $\mathcal{I}_{k}$ be the null space of the matrix $M_{f, k}$, that is, the space of $\tau \in \mathbb{C} \mathcal{T}_{k}$ with $f(\tau \cdot T)=0$ for each $k$-tangle $T$. Then $\mathcal{I}_{k}$ is an ideal in the algebra $\mathbb{C} \mathcal{I}_{k}$, and the quotient

$$
\mathcal{A}_{k}:=\mathbb{C} \mathcal{T}_{k} / \mathcal{I}_{k}
$$

is an algebra of dimension $\operatorname{rank}\left(M_{f, k}\right)$. We will indicate the elements of $\mathcal{A}_{k}$ just by their representatives in $\mathbb{C} \mathcal{T}_{k}$. Define the 'trace-like' function $\vartheta: \mathcal{A}_{k} \rightarrow \mathbb{C}$ by

$$
\vartheta(x):=f\left(x \cdot \mathbf{1}_{k}\right)
$$

for $x \in \mathcal{A}_{k}$. Then $\vartheta(x y)=\vartheta(y x)$ for all $x, y \in \mathcal{A}_{k}$ and $\vartheta\left(\mathbf{1}_{k}\right)=f(\wp)^{k}=n^{k}$.

We first show that $\mathcal{A}_{k}$ is semisimple. To this end, let for $k, m \in \mathbb{Z}_{+}$and $\pi \in S_{m}$, $P_{k, \pi}$ be the $k m$-tangle consisting of $k m$ disjoint edges $e_{i, j}$ for $i=1, \ldots, m$ and $j=1, \ldots, k$, where the head (sink) of $e_{i, j}$ is labeled $j+(i-1) m$ and its tail (root) is labeled $j+(\pi(i)-1) k$.

We also need a product $S \sqcup T$ of a $k$-tangle $S$ and an $l$-tangle $T$ : It is the $k+l$-tangle obtained from the disjoint union of $S$ and $T$ by adding $k$ to all labels in $T$. This product can be extended bilinearly to $\mathbb{C} \mathcal{T}_{k} \times \mathbb{C} \mathcal{T}_{l} \rightarrow \mathbb{C} \mathcal{T}_{k+l}$. The product is associative, so that for any $x \in \mathbb{C} \mathcal{T}_{k}$, the $m$-th power $x^{\sqcup m}$ is well defined.

Then for any $x \in \mathbb{C} \mathcal{T}_{k}$ and $\rho, \sigma \in S_{m}$, one has

$$
f\left(x^{\sqcup m} P_{k, \rho} \cdot P_{k, \sigma}\right)=f\left(x^{\sqcup m} \cdot P_{k, \rho} P_{k, \sigma}\right)=f\left(x^{\sqcup m} \cdot P_{k, \rho \sigma}\right)=\prod_{c} \vartheta\left(x^{|c|}\right),
$$


where $c$ ranges over the orbits of permutation $\rho \sigma$. We are going to use that for each $x \in \mathbb{C} \mathcal{T}_{k}$, the $S_{m} \times S_{m}$ matrix $\left(f\left(x^{\sqcup m} P_{k, \rho} \cdot P_{k, \sigma}\right)\right)_{\rho, \sigma \in S_{m}}$ has rank at most $\operatorname{rank}\left(M_{f, k m}\right)$ (since $x^{\sqcup m} P_{k, \rho}$ belongs to $\mathbb{C} \mathcal{T}_{k m}$, for each $\rho$ ).

Claim 1 For each $k$, if $x$ is a nilpotent element of $\mathcal{A}_{k}$, then $\vartheta(x)=0$.

Proof Suppose $\vartheta(x) \neq 0$ and $x$ is nilpotent. Then there is a largest $t$ with $\vartheta\left(x^{t}\right) \neq 0$. Let $y:=x^{t}$. So $\vartheta(y) \neq 0$ and $\vartheta\left(y^{s}\right)=0$ for each $s \geq 2$. By scaling, we can assume that $\vartheta(y)=1$.

Choose $m$ with $m !>n^{2 k m}$. By (17), we have, for any $\rho, \sigma \in S_{m}$,

$$
f\left(y^{\sqcup m} P_{k, \rho} \cdot P_{k, \sigma}\right)=\delta_{\rho, \sigma^{-1}},
$$

since $\vartheta\left(x^{|c|}\right)=0$ if $|c|>1$, implying that the product in (17) is 0 if $\rho \sigma \neq \mathrm{id}$.

So $\operatorname{rank}\left(M_{f, k m}\right) \geq m$ !, contradicting the fact that $\operatorname{rank}\left(M_{f, k m}\right) \leq n^{2 k m}<m$ !.

The following is a direct consequence of Claim 1:

Claim $2 \mathcal{A}_{k}$ is semisimple, for each $k$.

Proof As $\mathcal{A}_{k}$ is finite-dimensional, it suffices to show that for each nonzero element $x$ of $\mathcal{A}_{k}$, there exists $y$ with $x y$ not nilpotent. As $x \notin \mathcal{I}_{k}$, we know that $f(x \cdot y) \neq 0$ for some $y \in \mathcal{A}_{k}$. So $\vartheta(x y) \neq 0$, and hence, by Claim 1, xy is not nilpotent.

Claim 3 For each $k$, if $x$ is a nonzero idempotent in $\mathcal{A}_{k}$, then $\vartheta(x)$ is a positive integer.

Proof Let $x$ be any idempotent. Then for each $m \in \mathbb{Z}_{+}$and $\rho, \sigma \in S_{m}$, by (17):

$$
f\left(x^{\sqcup m} P_{k, \rho} \cdot P_{k, \sigma}\right)=\vartheta(x)^{o(\rho \sigma)},
$$

where $o(\pi)$ denotes the number of orbits of any $\pi \in S_{m}$. So for each $m$ :

$$
\operatorname{rank}\left(\left(\vartheta(x)^{o(\rho \sigma)}\right)_{\rho, \sigma \in S_{m}}\right) \leq \operatorname{rank}\left(M_{f, k m}\right) \leq f(\bigcirc)^{2 k m}
$$

This implies (cf. [13]) that $\vartheta(x) \in \mathbb{Z}$ and $\vartheta(x) \leq f(\bigcirc)^{k}$. As $\mathbf{1}_{k}-x$ also is an idempotent in $\mathcal{A}_{k}$ and as $\vartheta\left(\mathbf{1}_{k}\right)=f(\bigcirc)^{k}$, we have $f(\bigcirc)^{k} \geq \vartheta\left(\mathbf{1}_{k}-x\right)=f(\bigcirc)^{k}-\vartheta(x)$. So $\vartheta(x) \geq 0$.

Suppose finally that $x$ is nonzero, while $\vartheta(x)=0$. As $\vartheta(y) \geq 0$ for each idempotent $y$, we may assume that $x$ is a minimal nonzero idempotent. Let $J$ be the two-sided ideal generated by $x$. As $\mathcal{A}_{k}$ is semisimple and $x$ is a minimal nonzero idempotent, $J \cong \mathbb{C}^{m \times m}$ for some $m$, yielding a trace function on $J$. As $\vartheta$ is linear, there exists an $a \in J$ such that $\vartheta(z)=\operatorname{tr}(z a)$ for each $z \in J$. As $\vartheta(y z)=\vartheta(z y)$ for all $y, z \in J$, we have $\operatorname{tr}(z a y)=\operatorname{tr}(z y a)$ for all $y, z \in J$. So $a y=y a$ for all $y \in J$; hence, $a$ is equal to a scalar multiple of the $m \times m$ identity matrix in $J$.

As $x \neq 0, f(x \cdot y) \neq 0$ for some $y \in \mathcal{A}_{k}$, so $\vartheta(x y) \neq 0$. Hence, $a \neq 0$, and so $\vartheta(x) \neq 0$ (as $x$ is a nonzero idempotent), contradicting our assumption. 
As $\mathbf{1}_{1}$ is an idempotent in $\mathcal{A}_{1}$, Claim 3 implies that $f(\bigcirc)=\vartheta\left(\mathbf{1}_{1}\right)$ is a nonnegative integer, say $n$. Define an element $\Delta \in \mathbb{C} \mathcal{T}_{n+1}$ as follows. For $\pi \in S_{n+1}$, let $T_{\pi}$ be the $(n+1)$-tangle consisting of $n+1$ disjoint directed edges $e_{1}, \ldots, e_{n+1}$, where the head of $e_{i}$ is labeled $i$ and its tail is labeled $\pi(i)$, for $i=1, \ldots, n+1$. Then

$$
\Delta:=\sum_{\pi \in S_{n+1}} \operatorname{sgn}(\pi) T_{\pi}
$$

Then $(n+1) !^{-1} \Delta$ is an idempotent in $\mathbb{C} \mathcal{T}_{n+1}$, and

$$
\begin{aligned}
\vartheta(\Delta) & =\sum_{\pi \in S_{n+1}} \operatorname{sgn}(\pi) n^{o(\pi)}=\sum_{\pi \in S_{n+1}} \operatorname{sgn}(\pi) \sum_{\substack{\varphi:[n+1] \rightarrow[n] \\
\varphi \circ \pi=\varphi}} 1 \\
& =\sum_{\varphi:[n+1] \rightarrow[n]} \sum_{\substack{\pi \in S_{n+1} \\
\varphi \circ \pi=\varphi}} \operatorname{sgn}(\pi)=0,
\end{aligned}
$$

since $f(\mathcal{O})=n$ and since no $\varphi:[n+1] \rightarrow[n]$ is injective. So by Claim $3, \Delta=0$ in $\mathcal{A}_{n+1}$. That is, $\Delta \in \mathcal{I}_{n+1}$. So, by definition of $\mathcal{I}_{n+1}$,

$$
\Delta \cdot \mathbb{C} \mathcal{T}_{n+1} \subseteq \operatorname{Ker}(f)
$$

To conclude the proof of (iv) $\Longrightarrow$ (iii), we follow a line of arguments similar to that in [6]. Recall that $p: \mathbb{C C} \rightarrow \mathcal{O}\left(S^{2}(\mathfrak{g l}(n))\right)$ is defined by $p(C)(X):=p_{X}(C)$ for all $C \in \mathcal{C}$ and $X \in S^{2}(\mathfrak{g l}(n))$.

Claim 4 Ker $p \subseteq \Delta \cdot \mathbb{C} \mathcal{T}_{n+1}$.

Proof Let $\gamma \in \mathbb{C C}$ with $p(\gamma)=0$. We prove that $\gamma \in \Delta \cdot \mathbb{C} \mathcal{T}_{n+1}$. As each homogeneous component of $p(\gamma)$ is 0 , we can assume that $\gamma$ is a linear combination of multiloop chord diagrams that all have the same number $m$ of chords.

Let $H$ be the group of permutations of $[2 m]$ that maintain the collection $\{\{2 i-$ $1,2 i\} \mid i \in[m]\}$. Then $H$ naturally acts on $S_{2 m}$ by $\rho \cdot \pi:=\rho \pi \rho^{-1}$ for $\rho \in H$ and $\pi \in S_{2 m}$.

For any $\pi \in S_{2 m}$, let $C_{\pi}$ be the multiloop chord diagram with vertex set [ $2 m$ ], chords $\{2 i-1,2 i\}$ (for $i=1, \ldots, m)$ and directed edges $(i, \pi(i)$ ) (for $i=1, \ldots, 2 m$ ). Each multiloop chord diagram with $m$ chords is isomorphic to $C_{\pi}$ for some $\pi \in S_{2 m}$. Therefore, we can write $\gamma=\sum_{\pi \in S_{2 m}} \lambda(\pi) C_{\pi}$ with $\lambda: S_{2 m} \rightarrow \mathbb{C}$. As $H$ leaves any $C_{\pi}$ invariant up to isomorphism, we can assume that $\lambda$ is $H$-invariant.

Define linear functions $F_{\pi}$ (for $\pi \in S_{2 m}$ ) and $F$ on $\mathfrak{g l}(n)^{\otimes 2 m}$ by

$$
F_{\pi}\left(\left(a_{1} \otimes b_{1}\right) \otimes \cdots \otimes\left(a_{2 m} \otimes b_{2 m}\right)\right):=\prod_{i=1}^{2 m} b_{i}\left(a_{\pi(i)}\right) \text { and } F:=\sum_{\pi \in S_{2 m}} \lambda(\pi) F_{\pi},(24)
$$

for $a_{1}, \ldots, a_{2 m} \in \mathbb{C}^{n}$ and $b_{1}, \ldots, b_{2 m} \in\left(\mathbb{C}^{n}\right)^{*}$. Note that $F_{\pi}\left(R^{\otimes m}\right)=p\left(C_{\pi}\right)(R)$ for any $R \in S^{2}(\mathfrak{g l}(n))$. Hence, $F\left(R^{\otimes m}\right)=p(\gamma)(R)=0$. We show that this implies that $F=0$. 
Indeed, suppose $F\left(v_{1} \otimes \cdots \otimes v_{2 m}\right) \neq 0$ for some $v_{1}, \ldots, v_{2 m} \in \mathfrak{g l}(n)$. For each $x \in \mathbb{C}^{m}$, define

$$
R_{x}:=\sum_{i=1}^{m} x_{i}\left(v_{2 i-1} \otimes v_{2 i}+v_{2 i} \otimes v_{2 i-1}\right) \in S^{2}(\mathfrak{g l}(n))
$$

As $F$ is $H$-invariant (since $\lambda$ is $H$-invariant), the coefficient of $x_{1} \cdots x_{m}$ in the polynomial $F\left(R_{x}^{\otimes m}\right)$ is equal to $|H| \cdot F\left(v_{1} \otimes \cdots \otimes v_{2 m}\right) \neq 0$. So the polynomial is nonzero; hence, $F\left(R_{x}^{\otimes m}\right) \neq 0$ for some $x$, a contradiction. Therefore, $F=0$.

Next, define the following polynomials $q_{\pi}$ (for $\pi \in S_{2 m}$ ) and $q$ on $\mathbb{C}^{2 m \times 2 m}$ :

$$
q_{\pi}(X):=\prod_{i=1}^{2 m} X_{i, \pi(i)} \text { and } q:=\sum_{\pi \in S_{2 m}} \lambda(\pi) q_{\pi},
$$

for $X=\left(X_{i, j}\right) \in \mathbb{C}^{2 m \times 2 m}$. Then

$$
q(X)=0 \text { if } \operatorname{rank}(X) \leq n
$$

Indeed, if $\operatorname{rank}(X) \leq n$, then $X=\left(b_{i}\left(a_{j}\right)\right)_{i, j=1}^{2 m}$ for some $a_{1}, \ldots, a_{2 m} \in \mathbb{C}^{n}$ and $b_{1}, \ldots, b_{2 m} \in\left(\mathbb{C}^{n}\right)^{*}$. By $(24)$ and $(26), q\left(\left(b_{i}\left(a_{j}\right)\right)_{i, j=1}^{2 m}\right)=F\left(\left(a_{1} \otimes b_{1}\right) \otimes \cdots \otimes\right.$ $\left.\left(a_{2 m} \otimes b_{2 m}\right)\right)=0$, proving $(27)$.

By the Second Fundamental Theorem (SFT) of invariant theory for GL( $n$ ) (cf. [8] Theorem 12.2.12), (27) implies that $q$ belongs to the ideal in $\mathcal{O}\left(\mathbb{C}^{2 m \times 2 m}\right)$ generated by the $(n+1) \times(n+1)$ minors of $\mathbb{C}^{2 m \times 2 m}$. Since each monomial in $q(X)$ contains precisely one variable from each row of $X$ and precisely one variable from each column of $X$, this implies $\gamma \in \Delta \cdot \mathbb{C} \mathcal{T}_{n+1}$.

Claim 4 and (23) imply $\operatorname{Ker}(p) \subseteq \operatorname{Ker}(f)$, and so there exists a linear function $\varphi: p(\mathbb{C} C) \rightarrow \mathbb{C}$ such that $\varphi \circ p=f$. Then $\varphi$ is an algebra homomorphism, since for $C, D \in \mathcal{C}$ one has $\varphi(p(C) p(D))=\varphi(p(C D))=f(C D)=f(C) f(D)=$ $\varphi(p(C)) \varphi(p(D))$.

We now apply some more invariant theory. As before, $\operatorname{GL}(n)$ acts on $\mathfrak{g l}(n)$ by $h \cdot M:=h M h^{-1}$ for $h \in \mathrm{GL}(n)$ and $M \in \mathfrak{g l}(n)$. This action transfers naturally to $S^{2}(\mathfrak{g l}(n))$. By the FFT of invariant theory, we have

$$
\mathcal{O}\left(S^{2}(\mathfrak{g l}(n))\right)^{\mathrm{GL}(n)}=p(\mathbb{C} \mathcal{C}) .
$$

So $\varphi$ is an algebra homomorphism $\mathcal{O}\left(S^{2}(\mathfrak{g l}(n))\right)^{\mathrm{GL}(n)} \rightarrow \mathbb{C}$. Hence, the affine GL $(n)$ variety

$$
\mathcal{V}:=\left\{R \in S^{2}(\mathfrak{g l}(n)) \mid q(R)=\varphi(q) \text { for each } q \in \mathcal{O}\left(S^{2}(\mathfrak{g l}(n))\right)^{\mathrm{GL}(n)}\right\}
$$

is nonempty (as $\mathrm{GL}(n)$ is reductive). By (28) and by substituting $q=p(C)$ in (29),

$$
\mathcal{V}:=\left\{R \in S^{2}(\mathfrak{g l}(n)) \mid p_{R}=f\right\} .
$$


Hence, as $\mathcal{V} \neq \varnothing$ we have (14) (iii).

To show (14) (ii), by (11) it suffices to show that the function $\widehat{p}\left(\tau_{4}\right): S^{2}(\mathfrak{g l}(n)) \rightarrow$ $\mathfrak{g l}(n)^{\otimes 3}$ has a zero on $\mathcal{V}$. Suppose $\widehat{p}\left(\tau_{4}\right)$ has no zero in $\mathcal{V}$. Then by the Nullstellensatz, there exists a regular function $q: \mathcal{V} \rightarrow \mathfrak{g l}(n)^{\otimes 3}$ such that $\operatorname{tr}\left(\widehat{p}\left(\tau_{4}\right)(R) q(R)\right)=1$ for each $R \in \mathcal{V}$. Applying the Reynolds operator, we can assume, as $\widehat{p}\left(\tau_{4}\right)$ is $\operatorname{GL}(n)$ equivariant, that also $q$ is GL(n)-equivariant. Then by the FFT of invariant theory, $q=\widehat{p}(\tau)$ for some $\tau \in \mathbb{C} \mathcal{T}_{3}$. This gives $1=\operatorname{tr}\left(\widehat{p}\left(\tau_{4}\right) \widehat{p}(\tau)\right)=p\left(\tau_{4} \cdot \tau\right)$. However, by (14) (iii), $p\left(\tau_{4} \cdot \tau\right)(R)=f\left(\tau_{4} \cdot \tau\right)=0$ for some $R$, a contradiction. This proves (14) (ii).

Finally, to show (14) (i), choose $R$ in the (unique) closed GL( $n)$-orbit contained in $\mathcal{V}$ (cf. [3,11]). Then $\widehat{p}_{R}\left(\tau_{4}\right)=0$, since by (ii), $\mathcal{V}$ contains some $R^{\prime}$ with $\widehat{p}_{R^{\prime}}\left(\tau_{4}\right)=0$ and since $R$ belongs to the closed orbit. As $R$ belongs to $S^{2}(\mathfrak{g l}(n))$, we can write

$$
R=\sum_{i=1}^{k} b_{i} \otimes b_{i}
$$

for some linearly independent $b_{1}, \ldots, b_{k} \in \mathfrak{g l}(n)$. By $(11), b_{1}, \ldots, b_{k}$ form an orthonormal basis of a metrized Lie subalgebra $\mathfrak{g}$ of $\mathfrak{g l}(n)$.

We prove that the identity id $: \mathfrak{g} \rightarrow \mathfrak{g l}(n)$ is a completely reducible representation of $\mathfrak{g}$. Choose a chain $0=I_{0} \subset I_{1} \subset I_{2} \subset \cdots \subset I_{k-1} \subset I_{k}=\mathbb{C}^{n}$ of $\mathfrak{g}$-submodules of $\mathbb{C}^{n}$, with $k$ maximal. For each $j=1, \ldots, k$, choose a subspace $X_{j}$ such that $I_{j}=I_{j-1} \oplus X_{j}$. For each real $\lambda>0$, define $\Delta_{\lambda} \in \operatorname{GL}(n)$ by: $\Delta_{\lambda}(x)=\lambda^{j} x$ if $x \in X_{j}$.

Then for each $M \in \mathfrak{g}, M^{\prime}:=\lim _{\lambda \rightarrow \infty} \Delta_{\lambda} \cdot M$ exists. Indeed, if $x \in X_{j}$, then $M x \subseteq I_{j}$, and so $\lim _{\lambda \rightarrow \infty} \Delta_{\lambda} M \Delta_{\lambda}^{-1} x$ is equal to the projection of $M x$ on $X_{j}$, with respect to the decomposition $X_{1} \oplus \cdots \oplus X_{k}$ of $\mathbb{C}^{n}$. So $M^{\prime} X_{j} \subseteq X_{j}$ for all $j$.

Hence, by (31), also $R^{\prime}:=\lim _{\lambda \rightarrow \infty} \Delta_{\lambda} \cdot R$ exists and is equal to $\sum_{i=1}^{k} b_{i}^{\prime} \otimes b_{i}^{\prime}$. As $\operatorname{GL}(n) \cdot R$ is closed, there exists $h \in \mathrm{GL}(n)$ with $h^{-1} \cdot R=R^{\prime}$, i.e., $R=h \cdot R^{\prime}$. Hence, $\mathfrak{g}$ is spanned by $h \cdot b_{1}^{\prime}, \ldots, h \cdot b_{k}^{\prime}$. Therefore, $\mathfrak{g}=\left\{h \cdot M^{\prime} \mid M \in \mathfrak{g}\right\}$. Now $\left(h \cdot M^{\prime}\right) h X_{j}=h M^{\prime} X_{j} \subseteq h X_{j}$ for each $M \in \mathfrak{g}$ and $j$. So $M h X_{j} \subseteq h X_{j}$ for each $M \in \mathfrak{g}$ and $j$. Therefore, each $h X_{j}$ is a $\mathfrak{g}$-submodule. By the maximality of $k$, each $h X_{j}$ is irreducible, proving (14) (i).

Acknowledgments The research leading to these results has received funding from the European Research Council under the European Union's Seventh Framework Programme (FP7/2007-2013)/ERC Grant agreement number 339109.

Open Access This article is distributed under the terms of the Creative Commons Attribution 4.0 International License (http://creativecommons.org/licenses/by/4.0/), which permits unrestricted use, distribution, and reproduction in any medium, provided you give appropriate credit to the original author(s) and the source, provide a link to the Creative Commons license, and indicate if changes were made.

\section{References}

1. Bar-Natan, D.: Weights of Feynman diagrams and the Vassiliev knot invariants. see http://www.math. toronto.edu/ drorbn/papers (1991) 
2. Bar-Natan, D.: On the Vassiliev knot invariants. Topology 34, 423-472 (1995)

3. Brion, M.: Introduction to actions of algebraic groups. Les cours du C.I.R.M. 1, 1-22 (2010)

4. Chmutov, S., Duzhin, S., Mostovoy, J.: Introduction to Vassiliev Knot Invariants. Cambridge University Press, Cambridge (2012)

5. de la Harpe, P., Jones, V.F.R.: Graph invariants related to statistical mechanical models: examples and problems. J. Comb. Theory Ser. B 57, 207-227 (1993)

6. Draisma, J., Gijswijt, D., Lovász, L., Regts, G., Schrijver, A.: Characterizing partition functions of the vertex model. J. Algebra 350, 197-206 (2012)

7. Freedman, M.H., Lovász, L., Schrijver, A.: Reflection positivity, rank connectivity, and homomorphisms of graphs. J. Am. Math. Soc. 20, 37-51 (2007)

8. Goodman, R., Wallach, N.R.: Symmetry, Representations, and Invariants. Springer, Dordrecht (2009)

9. Kodiyalam, V., Raghavan, K.N.: Picture invariants and the isomorphism problem for complex semisimple Lie algebras. ArXiv http://arxiv.org/math/0402215v1 (2004)

10. Kontsevich, M.: Vassiliev's knot invariants. I.M. Gelfand seminar Part 2, Advances in Soviet Mathematics 16, American Mathematical Society, Providence, R.I., pp. 137-150 (1993)

11. Kraft, H.: Geometrische Methoden in der Invariantentheorie. Vieweg, Braunschweig (1984)

12. Lovász, L.: Large Networks and Graph Limits. American Mathematical Society, Providence, R.I. (2012)

13. Schrijver, A.: Characterizing partition functions of the vertex model by rank growth, preprint. ArXiv http://arxiv.org/abs/1211.3561 (2012)

14. Szegedy, B.: Edge coloring models and reflection positivity. J. Am. Math. Soc. 20, 969-988 (2007) 